\title{
Validación del test de troponinas en plasma a partir de una población sana. Una experiencia local.
}

Alberto Barría ${ }^{1}$, M. Jesús Vial ${ }^{2}$, Ana Tong ${ }^{2}$, Hernán Prat ${ }^{1}$, René Asenjo ${ }^{1}$.

1 Departamento de Cardiología, Hospital Clínico de la Universidad de Chile.

2 Laboratorio Central, Hospital Clínico de la Universidad de Chile.

Introducción: Las troponinas corresponden a proteínas estructurales del miocardiocito, su presencia en plasma se utiliza como marcador de injuria miocárdica. El test troponinas en plasma se utiliza actualmente para detectar daño miocárdico en pacientes en quienes se sospecha un infarto agudo al miocardio; para este propósito se toma como referencia valores de referencia validados en poblaciones de Inglaterra o Estados Unidos.

Objetivo: El propósito de este estudio obtener valores de referencia para nuestra población local.

Métodos y resultados: Se efectuó una determi- nación de troponina I en 500 sujetos sanos, se obtuvo el valor promedio de troponina I para esta población y el valor percentil 99 para esta población que se considera internacionalmente como el valor de referencia para determinar el límite normal, el valor P99 resultó significativamente menor en mujeres respecto a hombres.

Conclusión: En conclusión, se han obtenido valores de referencia para aplicar el test de troponina I en nuestro medio local.

Palabras claves: Infarto al miocardio, troponina I. 


\section{Validation of normal values for troponin I plasma levels in a Chilean population}

Background: Troponins are myocardiocyte proteins; their plasma level is used as a marker for myocardial injury. In the diagnosis of myocardial infarction values currently used as cut points are those validated elsewhere (USA, UK).

Aim: In this study we aimed to determine normal limits of Troponin I in a sample of Chilean subjects.

Methods and results: 500 healthy subjects had their troponin I levels measured, computing the mean and 99 percentile (p99) values. Both parameters were significantly lower in females compared to males (mean \pm SD $0.089 \pm 0.047$ vs $0.102 \pm 0.063, p<0.01$; p99 0.02 vs 0.03 , respectively).

Conclusion: These troponin I values may be used to assess the likelihood of myocardial infarction in Chilean subjects.

Keywords: Troponin I, Chilean population

\section{Introducción}

Las troponinas corresponden a proteínas fundamentales para la contracción del miocardio, ya que forman parte de la ultraestructura del aparato contráctil. Existen 3 clases de troponinas 1 : I, C y T; su función principal corresponde al acoplamiento excitación-contracción y regulación de ésta. Las isoformas T e I se encuentran también en el músculo estriado, pero son estructuralmente diferentes a las del miocardiocito ${ }^{2}$.

En la evaluación actual de un paciente en quien sospechamos un Síndrome Coronario Agudo (SCA), la presencia de troponinas libres en el plasma nos permite confirmar el diagnóstico y definir la presencia de daño miocárdico ${ }^{3-4}$. La detección de troponinas en plasma es posible a través de test serológicos que se basan en la detección de secuencias N-terminal de aminoácidos por anticuerpos específicos ${ }^{5}$. Existen otros test para detectar injuria miocárdica como la creatinkinasa y su fracción $\mathrm{MB}$, o la mioglobina; sin embargo, la pesquisa de troponinas en plasma ha demostrado mayor sensiblidad y especificidad 6 ; es el test serológico de elección para detección de injuria miocárdica de acuerdo a las Sociedades Americana ${ }^{7}$ y Europea de Cardiología ${ }^{8}$.

Se ha demostrado que la detección de troponinas en plasma traduce injuria miocárdica, y se correlaciona con la cantidad de masa miocárdica dañada ${ }^{9}$. Considerando la alta sensiblidad del test en la detección de injuria miocárdica, recientemente se ha perfeccionado la definición de infarto agudo al miocardio, considerándose actualmente: la presencia de síntomas compatibles y/o cambios electrocardiográficos, asociados a un alza del test de troponinas sobre el percentil 99 de la población ${ }^{\mathbf{1 0}}$.

El test de troponinas en plasma es frecuentemente utilizado en nuestro medio para detección de injuria miocárdica en pacientes que sospechamos SCA. Los valores de referencia utilizados, corresponden a aquellos validados para poblaciones anglosajonas ${ }^{11}$; no existen datos en la literatura local respecto a los valores normales para nuestra población. Las Sociedades internacionales de cardiología y bioquímica, así como la empresa que fabrica el test, recomiendan validarlo en la población en que será aplicado ${ }^{\mathbf{1 2}}$. El propósito de nuestro estudio es validarlo en nuestra población.

\section{Materiales y métodos}

El valor del test de troponinas que definimos como "sobre el normal de la población" corresponde por consenso a aquel que se ubica sobre el percentil 99* de una población normal. El propósito de nuestro estudio es buscar este valor en una población de sujetos sanos. La población elegida corresponde a 501 sujetos que acuden a nuestro centro como donantes de sangre voluntarios. Como screening, se realiza una encuesta de salud acerca de comorbilidades, patologías agudas y condiciones de riesgo para enfermedades de transmisión sexual. Aquellos sujetos en los cuales no se detectan condiciones de riesgo en la encuesta, se aceptan como donantes de sangre; estos fueron considerados como población sana.

A cada sujeto se le efectuó determinación de troponina I en plasma; el test utilizado corresponde al proporcionado por el laboratorio Johnson \& Johnson: "Vitros Inmunodiagnostic", y es el que está disponible en nuestro centro. La muestra de sangre utilizada correspondió a la misma en la cual se toma muestra para serología de enfermedades transmisibles, en consecuencia no requirió mayor volumen de sangre que el habitual en una 
donación. Una vez tomada la muestra fue procesada en el laboratorio central, el resultado de cada determinación fue almacenada en nuestra base de datos junto a las característica demográficas de cada sujeto.

Análisis de resultados: Se efectuó recolección de datos incluyendo edad, sexo, peso, presión arterial y frecuencia cardíaca al momento de la toma de muestra, valor de troponina I y grupo sanguíneo; los cuales fueron guardados en una planilla de Excel Microsoft. Para el cálculo del percentil 99, se ordenaron los valores de troponina de menor a mayor y se determinó el valor que dejó bajo el $99 \%$ de la distribución de los datos usando la función percentil; esto se efectuó para todo el grupo y de manera independiente para sujetos de sexo masculino y femenino. Posteriormente, se efectuó análisis de datos en programa STATA 7.0. Los resultados se expresaron como promedios, medianas y desviación estándar, según correspondía para cada característica analizada. Se evaluó la existencia de diferencias significativas entre los valores de troponinas según sexo; para esto se utilizó el test de diferencia de medias para comparar dos promedios de dos muestras independientes. Se evaluó la existencia de correlación entre el peso o edad y el nivel de troponinas mediante el coeficiente de correlación lineal de Pearson.

Los autores declaran no tener conflictos de intereses con la empresa que ha facilitado el test para la medición de troponina.

\section{Resultados}

Se efectuó determinación de troponinas en plasma a 501 sujetos sanos, de los cuales 58,5\% correspondieron a varones y $41.5 \%$ a mujeres; la edad promedio fue $32.5 \pm 11.8$ años con una mediana de 31 años. El peso promedio fue $73 \pm 13.3 \mathrm{~kg}$. La presión arterial sistólica promedio fue de $122.3 \pm 62.7 \mathrm{mmHg}$ y la diastólica promedio $72.1 \pm 9.8 \mathrm{mmHg}$. El grupo sanguíneo correspondió en un $55.3 \%$ al O y en $31.7 \%$ al A, $10.9 \%$ al B y $2 \%$ el grupo AB. (Tabla 1 ).

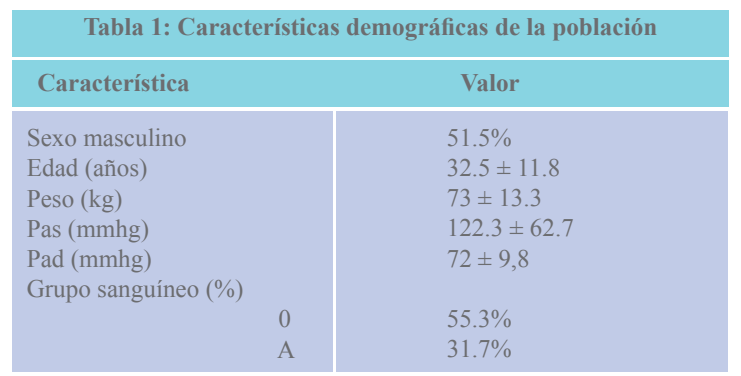

La media de las troponinas en la población fue $0.096 \mathrm{ng} /$ $\mathrm{ml}$ y el percentil 99 fue de $0.026 \mathrm{ng} / \mathrm{ml}$. En los hombres, el percentil 99 fue $0.03 \mathrm{ng} / \mathrm{ml}$ y en las mujeres fue 0.02 $\mathrm{ng} / \mathrm{ml}$. Esta diferencia fue significativa $(\mathrm{p}=0.0051)$. En la Tabla 2 se muestran los intervalos de confianza para el promedio del valor de troponinas en ambos sexos.

No existió correlación de la edad y tampoco del peso con el nivel de troponinas en plasma.

\begin{tabular}{|c|c|c|c|}
\hline Característica & n & Media \pm DE & IC $95 \%$ \\
\hline Mujeres & 208 & $0.089 \pm 0.047$ & $0.083-0.095$ \\
\hline Hombres & 293 & $0.102 \pm 0.063$ & $0.095-0.110$ \\
\hline
\end{tabular}

\section{Discusión}

La determinación de Troponinas en plasma corresponde a un test fundamental en la evaluación de pacientes en quienes se sospecha $\mathrm{SCA}^{\mathbf{1 3}}$. El valor del P99 para el test de troponinas en la población sana resultó $0.026 \mathrm{ng} / \mathrm{ml}$, menor que el valor de 0.034 propuesto por el fabricante. Este hallazgo puede variar la conducta respecto a considerar positiva una medición y aumentar el número de pacientes que caen en la categoría de infarto agudo al miocardio. Al dividir el grupo según género, el valor P99 resultó significativamente mayor en hombres que en mujeres. Si lo anterior se considera válido, sería necesario adoptar estos valores de $0,02 \mathrm{pg} / \mathrm{ml}$ en mujeres y de $0,03 \mathrm{pg} / \mathrm{ml}$ en hombres como punto de corte para injuria miocárdica; de lo contrario existe riesgo de subdiagnosticar un número no determinado de pacientes con injuria miocárdica y alto riesgo coronario. Con los datos que disponemos, no es clara la causa de la diferencia de valor P99 entre hombres y mujeres; podemos mencionar que esta diferencia es reportada cuando se efectúa validación de test de creatina kinasa y su fracción MB. Tampoco es clara la traducción clínica de esta diferencia, para lo cual será de utilidad evaluar el comportamiento de pacientes que cursen síndrome coronario agudo y tengan valor de troponina entre 0.020 y $0.029 \mathrm{pg} / \mathrm{ml}$, rango de valores que ha sido considerado normal hasta el momento.

Destacamos la ausencia de correlación entre el valor de troponinas en plasma con el peso o con la edad de los sujetos evaluados. Tomando en cuenta esto, podemos utilizar el test con seguridad en pacientes añosos u obesos. 
Este estudio corresponde a la primera experiencia reportada en nuestro medio para validación del test de troponinas en plasma; además de la utilidad clínica, los valores obtenidos se podrán utilizar para realizar investigación clínica en relación a marcadores de daño miocárdico, lo cual requiere validación previa del test. Interrogantes por responder corresponden al comportamiento de las troponinas en plasma en situaciones que aumentan el riesgo cardiovascular, como la insuficiencia renal, los accidentes cerebrovasculares, la hipertensión pulmonar y la sepsis severa ${ }^{\mathbf{1 4}}$.
Limitaciones: El grupo de estudio fue considerado sano, pero ello se basó, exclusivamente, en la encuesta al momento de donar sangre. Sin embargo, en la validación original del test de troponinas la condición de sano se basó también en una encuesta de salud.

Otro de los puntos débiles consiste en que el promedio de edad de nuestra población fue de $32.5 \pm 11.8$ años, considerablemente menor que la edad habitual, 60 a 70 años, de los pacientes coronarios Sin embargo, esta limitación pudiera no ser importante, dada la ausencia de correlación significativa entre valor de troponinas y edad, demostrada en este estudio.

\section{Referencias:}

1. OPIE L. Mechanisms of Cardiac Contraction and Relaxation. En Brauwald's Heart Disease. A Textbook of Cardiovascular Medicine. Philadephia; Saunders- Elsevier, 2008. 509- 560.

2. STACY M, MILENIO J, TANASIJEVIC, JAROLIM P. CARDIAC Troponin Assays: A View From the Clinical Chemistry Laboratory. Circulation 2007; 116: 501- 504.

3. LABUGGER R, ORGAN L, COLLIER C, ATAR D, VAN EYK J. Extensive Troponin I and T Modification Detected in Serum From Patients With Acute Miocardial Infarction. Circulation 2000; 102: 1221- 1226.

4. MADSEN L, CHISTENSEN G, LUND T, SEREBRUANY V, GRANGER C, HOEN I, et al. Time Course of Degradation of Cardiac Troponin I in Patients With Acute ST-Elevation Miocardial Infarction: The ASSENT-2 Troponin Substudy. Circulation Research 2006; 99: 1141- 1147.

5. APPLE S, JESSE R, NEWBY K, WU A, CHRISTENSON R, CANNON C, et al. National Academy of Clinical Biochemistry and IFCC Comittee for Standarization of Markers of Cardiac Damage Laboratory Medicine Practice Guidelines: Analitical Issues for Biochemical Markers of Acute Coronary Syndromes. Clinical Chemistry 2007; 53: 547- 551 .

6. ADAMS J, BODOR G, DAVILA-ROMAN V, DELMEZ J, APPLE F, LADENSON J, et al. Cardiac Troponin I. A marker with high specificity for cardiac injury. Circulation 1993; 88: 101- 106

7. ANTMAN E, ANBE D, WAYNE P, BATES E, GREEN L. HAND M, et al. ACC/AHA Guidelines for the Management of
Patients With ST-Elevation Miocardial Infarction. 2004 by the American College of Cardiology Foundation and the American Heart Association.

8. VAN DER WERFF,ARDISSINO D, BETRIU A,COKKINOS D, FALK E, FOX K, et al. Management of acute miocardial infarction in patients presenting with ST elevation. Eur Heart J 2003; 24: 28- 66 .

9. STEEN H, GIANNITSIS E, FUTTERER S, MERTEN C, JUENGER C, KATUS H. Cardiac Troponin T at 96 Hours After Acute Miocardial Infarction Correlates With Infarct Size and Cardiac Function. J Am Coll Cardiol 2006; 48: 2192- 2194.

10. THYGESEN C,ALPERT J, WHITE H. Universal Definition on Miocardial Infarction. Eur Heart J 2007; 28: 2525- 2538.

11. UETTWILLER-GEIGER D, WU A, APPLE F, JEVANS A, VENGE P, OLSON M, et al. Multocenter Evaluation of an Automated Assay for Troponin I. Clinical Chemistry 2002; 48: 869- 876.

12. APPLE F, QUIST H, DOYLE P, OTTO A, MURAKAMY M. Plasma 99th Percentile Reference Limits for Cardiac Troponin and Creatine Kinase MB Mass for Use with European Society of Cardiology/ American College of Cardiology Consensus Recomendations. Clinical Chemistry 2003; 49: 1331- 1136

13. NEWBY L, CHRISTENSON R, OHMAN E, ARMSTRONG P, THOMPSON T, LEE K, et al. Value of Serial Troponin T Measures for Early and Late Risk Stratification in Patients With Acute Coronary Syndromes. Circulation 1998; 98: 1853-1859

14. HAMM C, GIANNITSIS E, KATUS H. Cardiac Troponin Elevations in Patients Without Acute Coronary Syndrome. Circulation 2002; 106: 2871- 2872. 